\title{
EuNetAir Air Quality Joint-Exercise Intercomparison: Assessment of Microsensors Versus Reference Methods
}

\author{
C. Borrego ${ }^{1}$, A. M. Costa ${ }^{2}$, J. Ginja' 2 M. Amorim ${ }^{2}$ \\ ${ }^{1}$ IDAD; Dept. of Environment and Planning, University of Aveiro, Portugal \\ ${ }^{2}$ IDAD, Aveiro, Portugal, \\ cborrego@ua.pt
}

\author{
K. Karatzas ${ }^{3}$ \\ ${ }^{3}$ Environmental Informatics Research Group, Aristotle University, Thessaloniki, Greece \\ kkara@eng.auth.gr
}

\begin{abstract}
The $1^{\text {st }}$ EuNetAir Air Quality Joint-Exercise Intercomparison organized in Aveiro (Portugal) from 13-27 October 2014 focused on the evaluation and assessment of environmental gas/PM and meteorology microsensors versus standardised air quality referenced methods through an experimental urban air quality monitoring campaign. The IDAD Air Quality Mobile Laboratory was placed at an urban traffic location in Aveiro city centre to conduct continuous measurements with standardized equipment and referenced analysers of the following variables: $\mathrm{CO}, \mathrm{NO}_{x}, \mathrm{O}_{3}, \mathrm{SO}_{2}, \mathrm{PM}_{10}, \mathrm{PM}_{2.5}$, $\mathrm{BTEX}$, temperature, humidity, wind velocity/direction, solar radiation and precipitation. The comparison of the sensor data generated by different sensor-systems installed side-by-side with reference analysers will contribute to assess behaviour and the accuracy of low-cost sensor-systems in the real-world context as indicative measurements as defined by the Ambient Air Quality EU Directive 2008/50/EC while it will help in their calibration and further development.
\end{abstract}

Key words: Air quality, monitoring, reference methods, microsensors, experimental campaign

\section{Introduction}

The increasing trend of global urbanization and ub the production and freight of goods results in high levels of air pollutants in urban areas, decreasing air quality. Air quality monitoring and air pollution control is needed to safeguard human health and quality of life by implementing abatement strategies and stimulating environmental awareness among citizens. For this purpose, there are several techniques and technologies that can be used to monitor air pollution [1]. Thus, the use of microsensor networks can be seen as an innovative tool for future applications on air quality monitoring. Adopting versatile, mobile and low-cost methods for robust real time environmental surveillance can represent a solution to overcome the lack of small geographical scale air quality information [2]. However, despite the fact that air quality sensors performance allows for a paradigm shift in environmental monitoring, resulting in real time data generation, low operating costs and high efficiency, much research remains to be done in order to integrate these new technologies, particularly on the quality check of the sensors performance against conventional methods in field exercises [3, 4].

\section{Description of the experiment}

The COST Action TD1105 EuNetAir is a running networking (2012-2016) funded in the framework European Cooperation in the field of Scientific and Technical Research (COST) that addresses New Sensing Technologies for AirPollution Control and Environmental Sustainability. The $1^{\text {st }}$ EuNetAir Air Quality Joint-Exercise Intercomparison was organized in the scope of COST TD1105 to evaluate the performance and assess the different environmental gas/particulate matter microsensors versus standardized air quality referenced methods. This experimental urban air quality monitoring campaign was organized by IDAD - Institute of Environment and Development in Aveiro (Portugal) on 13-27 October 2014. The two-week experimental campaign was conducted in an urban traffic location in Aveiro city centre counting with 15 teams from research centres, universities and companies coming from 12 COST Countries. 


\section{Characterization of the study area}

The city of Aveiro is located in the central region of Portugal $\left(408^{\prime} \mathrm{N}, 8^{\circ} 39^{\prime} \mathrm{W}\right)$, with around 18756 citizens and a total area of approximately $8 \mathrm{~km}^{2}[5]$.

Aveiro presents a Mediterranean climate with an annual average temperature around $15^{\circ} \mathrm{C}$, and a daily variation amplitude between $5^{\circ} \mathrm{C}$ and $10^{\circ} \mathrm{C}$ for every month of the year. The annual averages of relative humidity varies from $79 \%-88 \%$.

Road traffic is the most significant source of emissions to the atmosphere in the city centre, despite the presence of an industrial area located at $10 \mathrm{~km}$ from the city centre.

\section{Sampling and data analysis}

The microsensors systems of 14 teams were installed side-by-side at IDAD Air Quality Mobile Laboratory that was equipped with standardized equipment and referenced analysers of the following variables: $\mathrm{CO}$ (non-dispersive infrared spectroscopy - EN14626), $\mathrm{NO}_{x}$ (chemiluminescence - EN 14211), $\mathrm{O}_{3}$ (ultraviolet photometry - EN 14625), $\mathrm{SO}_{2}$ (ultraviolet fluorescence - EN 14212), $\mathrm{PM}_{10}$ and $\mathrm{PM}_{2.5}$ (Beta-ray absorption method - ISO 10473 equivalent method), benzene (gas chromatography - EN 14662), temperature, humidity, wind velocity/direction, solar radiation, precipitation.

In this preliminary assessment from a total of 15 teams that participated in the campaign only results from 7 teams were analysed. A preliminary statistical analysis of field results was conducted allowing to assess the performance of different types of microsensors, namely metal oxide semiconductor sensors (MOS), electrochemical sensors and optical particle counter sensors (OPC).

The conversion process of microsensors raw data to concentration units was conducted by each participating team after becoming available the results from the reference methods.

\section{Results and discussion}

The analysis of data obtained with the reference methods shows that no exceedances to the limit values for $\mathrm{CO}, \mathrm{NO}_{2}, \mathrm{O}_{3}, \mathrm{SO}_{2}$ or benzene were recorded. However, over the two-week campaign, the $\mathrm{PM}_{10}$ daily limit of 50 $\mu \mathrm{g} \cdot \mathrm{m}^{-3}$ for the protection of human health was exceeded 6 times (from the $20^{\text {th }}$ to the $25^{\text {th }}$ of October). This was due to the associated traffic emissions and meteorological conditions and also to the simultaneous occurrence of natural events with the transport of particles from North Africa [6]. It was also noticeable that in the first week of the experimental campaign it was observed long periods of precipitation (total of $75.4 \mathrm{~mm}$ ), high relative humidity (average: $79 \%$, range: $44-90 \%$ ) and strong wind (average: 2.2 $\mathrm{m} . \mathrm{s}^{-1}$, range: $0.1-5.6 \mathrm{~m} . \mathrm{s}^{-1}$ ). For the second week the meteorological conditions changed with no periods of precipitation, high temperatures (average: $21^{\circ} \mathrm{C}$, range: $15-30^{\circ} \mathrm{C}$ ), lower relative humidity (average: 65\%, range: $39-87 \%$ ) and wind velocities (average $0.6 \mathrm{~m} . \mathrm{s}^{-1}$, range: $0.1-1.5 \mathrm{~m} . \mathrm{s}^{-1}$ ).

Figure 1 presents an example of correlations between data from microsensors and reference analysers versus data collection efficiency (expressed as a percentage etc) for different pollutants $\left(\mathrm{NO}_{2}, \mathrm{CO}, \mathrm{O}_{3}, \mathrm{PM}_{2.5}\right.$ and $\left.\mathrm{PM}_{10}\right)$. Each marker represents a sensor from a specific team, except for $\mathrm{CO}$ pollutant where 2 of the 3 MOS sensors belong to the same team.

Ideally microsensors should have strong correlations and high data collection efficiency throughout the entire duration of the campaign, meaning that they should be represented in the second quarter of the graphs area. However, some problems were identified concerning data collection efficiency, that may be related to the high relative humidity and temperatures during the campaign, intermittent communication failures and also the instability and crossreactivity caused by interfering gases.

The analysis shows that for $\mathrm{NO}_{2}$ and $\mathrm{CO}$ measurements performed with electrochemical and MOS sensors a stronger correlation with the reference method and a higher efficiency collecting data is noticed for electrochemical sensors. On the other hand, a greater correlation with the reference method and a higher efficiency in data collection is observed for $\mathrm{O}_{3}$ measurements resulting from MOS sensors. For NO measurements none of the sensors present the ideal behaviour since none of them is represented in the first quadrant of Figure 1. One of the NO electrochemical microsensors presents a high data collection efficiency $(n \approx 90 \%)$ and a very weak correlation with the reference method $\left(R^{2} \approx 0\right)$ despite the other presents a weak data collection efficiency $(n \approx 40 \%)$ and a very strong correlation with the reference method $\left(R^{2}=0.83\right)$. This difference may be attributed to the use of different materials in the development of these sensors or the application of different data processing algorithms developed by each of the teams. The OPC sensors for $\mathrm{PM}_{10}$ and $\mathrm{PM}_{2.5}$ present correlations that vary between $0.45-0.87$ and data collection efficiencies in the range of 67 $80 \%$. 

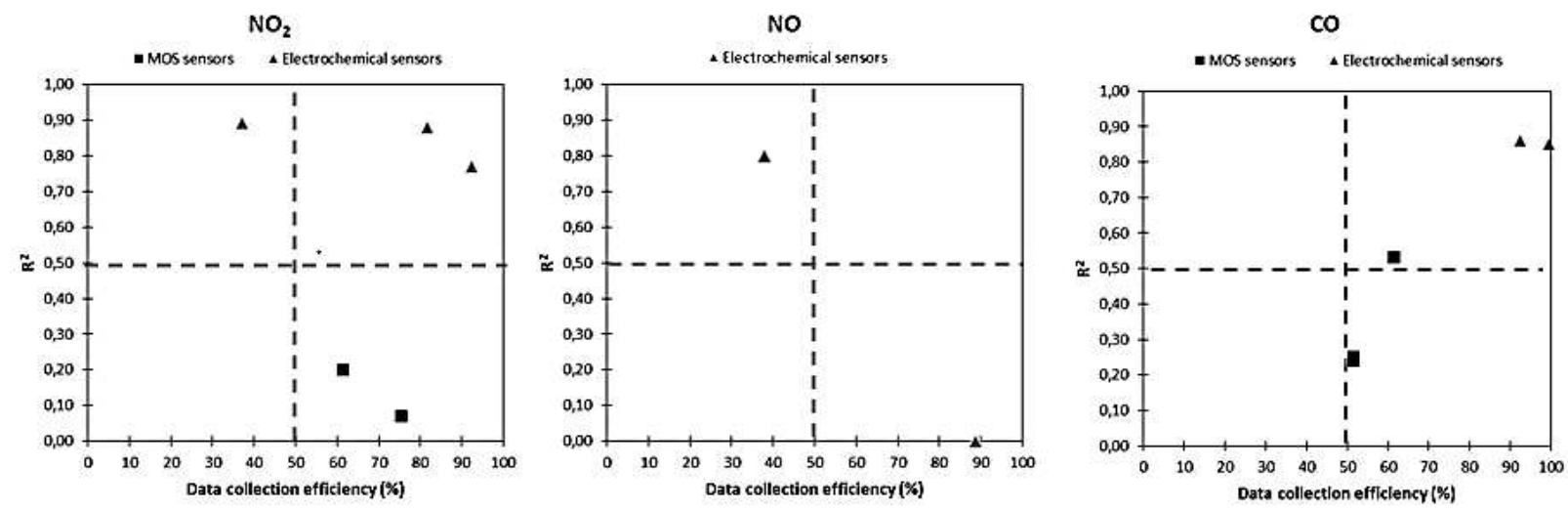

$\mathrm{O}_{3}$
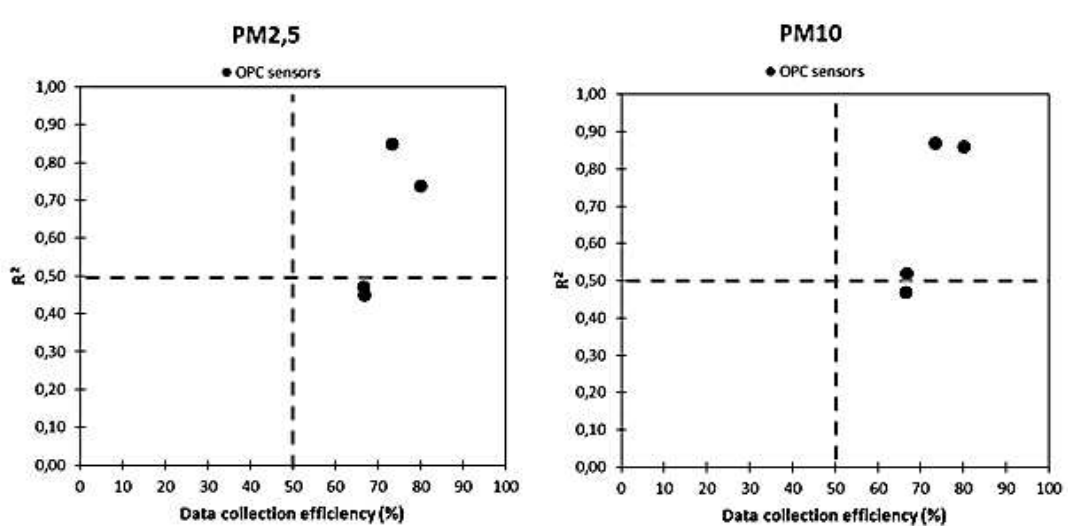

Fig. 1. Correlation between microsensors and reference analysers vs. data collection efficiency.

RStudio software was used in order to analyse the possible correlations of meteorological parameters in the microsensors measurement absolute error. The interferences were analysed for each team through correlation matrixes that show scatterplots between pairs of parameters (upper matrix plots) and their Pearson correlation coefficients (lower matrix values) for hourly values of the measured pollutants, temperature $(\mathrm{T})$ in ${ }^{\circ} \mathrm{C}$, relative humidity in \% $(\mathrm{RH})$, absolute humidity $\left(\mathrm{H}_{2} \mathrm{O}\right)$ in $\mathrm{mg} \cdot \mathrm{m}^{-3}$, solar radiation (rad) in $\mathrm{W} \cdot \mathrm{m}^{-2}$, wind velocity $(\mathrm{WV})$ in $\mathrm{m} . \mathrm{s}^{-1}$ and pressure in $\mathrm{hPa}(\mathrm{P})$.

Figure 2 presents an example of a correlation matrix with meteorological parameters in the measurement error for two MOS sensors from the same team.

According to Figure 2 the $\mathrm{O}_{3}$ microsensor measurement error shows weak correlations with all the meteorological parameters, suggesting that these parameters do not interfere with the readings performed by the equipment.

The measurement error of $\mathrm{NO}_{2}$ sensor demonstrated a moderate correlation with the wind speed and the atmospheric pressure, presenting Pearson correlation coefficients of 0.56 and -0.55 , associated to high wind speed and low pressures respectively.

The measurement error of $\mathrm{CO}$ sensors showed weak correlations with the meteorological parameters. The highest correlation coefficient found was -0.33 resulting from the measurement error of $\mathrm{CO}$ sensor and the absolute humidity.

Taking into consideration the results obtained for all teams, it can be concluded that MOS sensors exhibit a lower correlation with the respective reference method. Also presented a higher cross-sensitivity of meteorological parameters, demonstrating a high sensitivity to the presence of other compounds and low selectivity towards the target gas. 


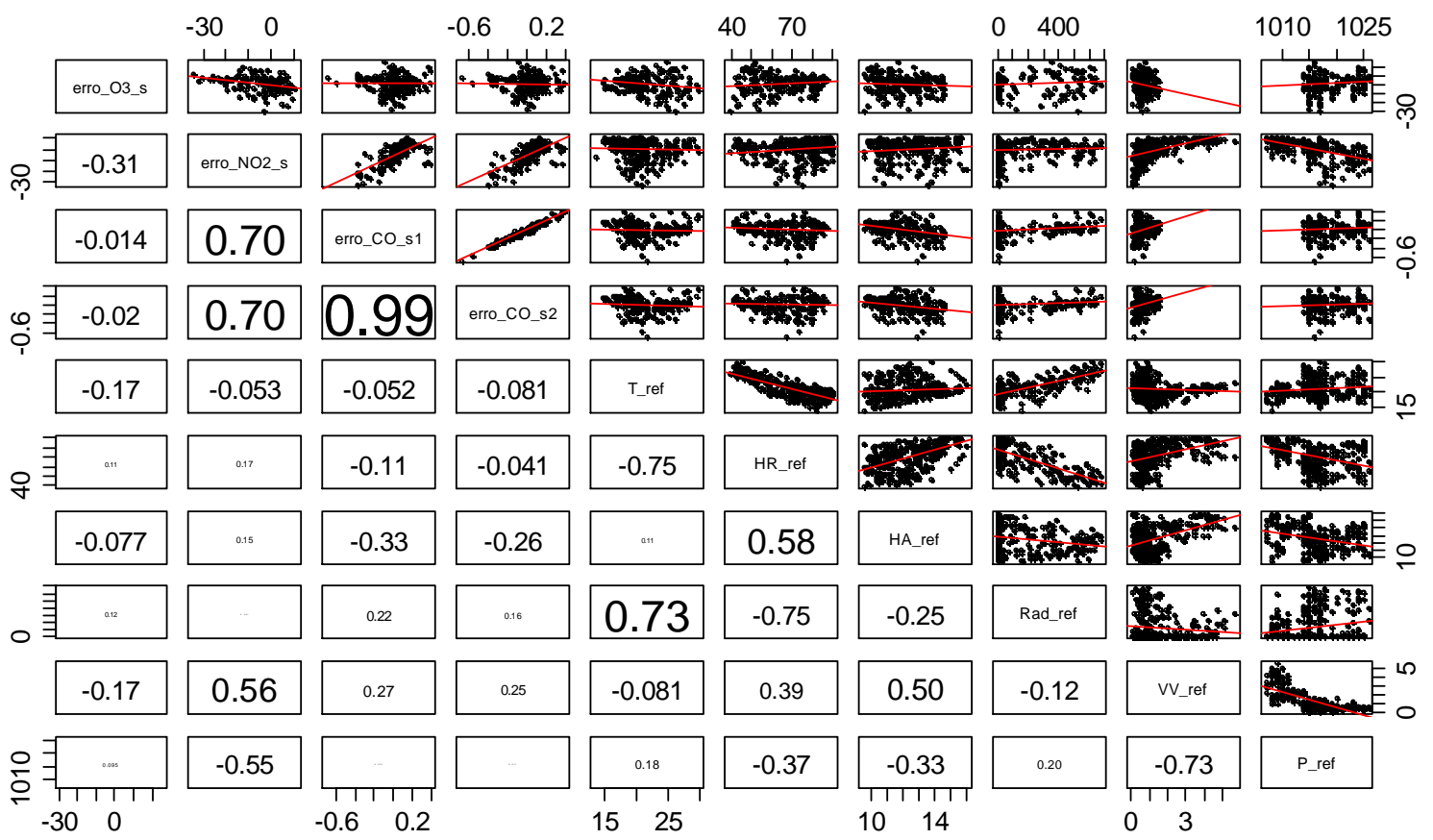

Fig. 2. Correlation matrix of the measuring error of MOS microsensors and the hourly reference values of meteorological parameters, $\mathrm{T}$ in ${ }^{\circ} \mathrm{C}, \mathrm{RH}$ in \%, $\mathrm{H}_{2} \mathrm{O}$ in $\mathrm{H} \mathrm{H}_{2} \mathrm{O} . \mathrm{m}^{-3}$, rad in W.m. ${ }^{-2}$, WS in $\mathrm{m}^{-1} \mathrm{~s}^{-1}$ and $\mathrm{P}$ in $\mathrm{hPa}$.

\section{Conclusions}

The use of microsensors for regulatory purposes is still not mentioned in European legislation, nevertheless the real-time collected data combined with standardized monitoring have an enormous potential to be applied in new strategies for air quality control, rapid mapping of air pollution over small areas, validation of atmospheric dispersion models or evaluation of population exposure, and for the development of user-tailored, geo-referenced, human centred, quality of life information services.

The preliminary evaluation of the $1^{\text {st }}$ EuNetAir campaign results shows that microsensors can be a promising technique for air quality monitoring but it is still necessary to establish an evaluation protocol approaching issues as sensitivity, selectivity (known interferences), short and long term stability, model equation and data validation, as well as calibration [7].

This joint experimental campaign must be seen as a first step to the research and development of low-cost sensors for pollutants monitoring, contributing for the evaluation of sensor performance in field exercises. Nevertheless data treatment of sensor signals and cross sensitivity studies still remains a challenging work for the future.

\section{Acknowledgements}

The authors would like to acknowledge the support of COST Action TD1105 - European
Network on New Sensing Technologies for AirPollution Control and Environmental Sustainability - EuNetAir.

\section{References}

[1] M. Penza, D. Suriano, M.G. Villani, L. Spinelle, M Gerboles, Towards air quality indices in smart cities by calibrated low-cost sensors applied to networks. IEEE SENSORS 2014 Proceedings, 2012-2017 (2014); doi:10.1109/ICSENS.2014.6985429

[2] P. Kumar, L. Morawska, C. Martani, G. Biskos, M. Neophytou, S. Di Sabatino, M. Bell, L. Norford, R. Britter, The rise of low-cost sensing for managing air pollution in cities. Environment International 75, 199205 (2015); doi:10.1016/j.envint.2014.11.019

[3] M. Aleixandre, M. Gerboles, Review of Small Commercial Sensors for Indicative Monitoring of Ambient Gas, Chemical Engineering Transactions 30, 169-174 (2012); doi: 10.3303/CET1230029.

[4] M.I. Mead, O. a. M. Popoola, G.B. Stewart, P. Landshoff, M. Calleja, M. Hayes, J.J. Baldovi, M.W. McLeod, T.F. Hodgson, J. Dicks, A. Lewis, J. Cohen, R. Baron, J.R. Saffell, R.L. Jones, The use of electrochemical sensors for monitoring urban air quality in low-cost, high-density networks, Atmospheric Environment 70, 186-203 (2013); doi:10.1016/j.atmosenv.2012.11.06.

[5] INE, Instituto Nacional de Estatística I.P., Censos 2011 Resultados Definitivos - Região Centro. Lisboa, (2012). ISBN: 978-989-25-0184-0.

[6] Portuguese Environment Agency (APA): http://www.apambiente.pt/index.php?ref $=16 \&$ subref $=82$ \&sub2ref $=316 \&$ sub3ref $=941$ (consulted in July 2015)

[7] L. Spinelle, M. Aleixandre, M. Gerboles, Protocol of evaluation and calibration of low-cost gas sensors for the monitoring of air pollution, Publications Office of the European Union, (2013) EUR 26112 EN. 